\title{
Large-scale dynamics of sandy coastlines: Diffusivity and instability
}

\author{
A. Falqués and D. Calvete \\ Applied Physics Department, Universitat Politècnica de Catalunya, Barcelona, Catalonia, Spain \\ Received 12 July 2004; revised 14 October 2004; accepted 24 November 2004; published 8 March 2005.
}

[1] The dynamics of small-amplitude perturbations of an otherwise rectilinear coastline due to the wave-driven alongshore sediment transport is examined at large time and length scales (years and kilometers). A linear stability analysis is performed by using an extended one-line shoreline model with two main improvements: (1) the curvature of the coastline features is accounted for and (2) the coastline features are assumed to extend offshore as a bathymetric perturbation up to a finite distance. For high incidence angles, instability is found in accordance with Ashton et al. (2001). However, it is seen that instability is inhibited by high waves with long periods and gently sloping shorefaces so that in this case the coastline may be stable for any angle. Similarly, there is no instability if the bathymetric perturbation is confined very close to the coast. It is found that the traditional linearized one-line model (Larson et al., 1987) tends to overpredict the coastline diffusivity. The overprediction is small for the conditions leading to a stable coastline and for moderate incidence angles but can be very dramatic for the conditions favoring instability. An interesting finding is that high-angle waves instability has a dominant wavelength at the linear regime, which is in the order of $4-15 \mathrm{~km}$, one to two orders of magnitude larger than the length scale of surf zone rhythmic features. Intriguingly, this is roughly the same range of the wavelength of some observed shoreline sand waves and, in particular, those observed along the Dutch coast. A model application to this coast is presented.

Citation: Falqués, A., and D. Calvete (2005), Large-scale dynamics of sandy coastlines: Diffusivity and instability, J. Geophys. Res., 110, C03007, doi:10.1029/2004JC002587.

\section{Introduction}

[2] In spite of the complexity of 3D nearshore morphodynamics, the so-called one-line modeling has had some success in understanding and predicting the dynamics of sandy coastlines at large space and timescales [PelnardConsidère, 1956; Horikawa, 1988; Komar, 1998]. This is a severe simplification and consists in averaging on the vertical and the cross-shore directions so that the morphodynamical active region collapses in a single line which represents the coastline. The changes in coastline position are then given by convergence/divergence of the total alongshore sediment transport rate $Q$ which is determined just by the wave forcing without account of surf zone hydrodynamics (water inertia, mass conservation, etc.). Cross-shore sediment transport is usually not explicitly considered. It is however always implicit to ensure the sediment redistribution that is necessary to reach the equilibrium beach profile after the changes which are driven by alongshore transport. Despite all those simplifications, the one-line modeling has been used for years, specially by coastal engineers and has proven to have reasonable skill for coastline evolution prediction at large time- and space scales

Copyright 2005 by the American Geophysical Union. 0148-0227/05/2004JC002587\$09.00 (years and kilometers) [Larson et al., 1987; Larson and Kraus, 1991].

[3] The sediment transport rate $Q$ at each position along the coast depends on the angle between wave fronts in deep water and the local coastline orientation, $\alpha_{\infty}$ [Komar, 1998; Horikawa, 1988] (see Figure 1). The transport rate rises from zero at normal wave incidence up to a maximum for $\alpha_{\infty} \simeq \alpha_{c}$ and drops to zero again for $\alpha_{\infty}=90^{\circ}$. It was shown by Ashton et al. [2001; see also Murray et al., 2001] that the existence of such a maximum, which occurs for $\alpha_{c} \simeq 42^{\circ}$, renders a rectilinear coastline unstable when the incidence angle is larger than $\alpha_{c}$. To this end, Ashton et al. [2001] used a nonlinear cellular model based on the one-line concept. They found that if $\alpha_{\infty}>\alpha_{c}$ any small perturbation of the rectilinear coastline grows. Nonlinear effects make the largest perturbations eventually dominate so that largescale coastline patterns resembling shoreline sand waves, capes and spits emerge. In general, their wavelength is not fixed but grows with time.

[4] On the other hand, the existence of the "high-angle waves instability" has some implications for the traditional one-line coastline modeling whose linearized governing equation is a diffusion equation [Pelnard-Considère, 1956]. For small amplitude perturbations, the formulation of Ashton et al. [2001] leads to a diffusion equation too. However, the diffusivity in the traditional approach is 


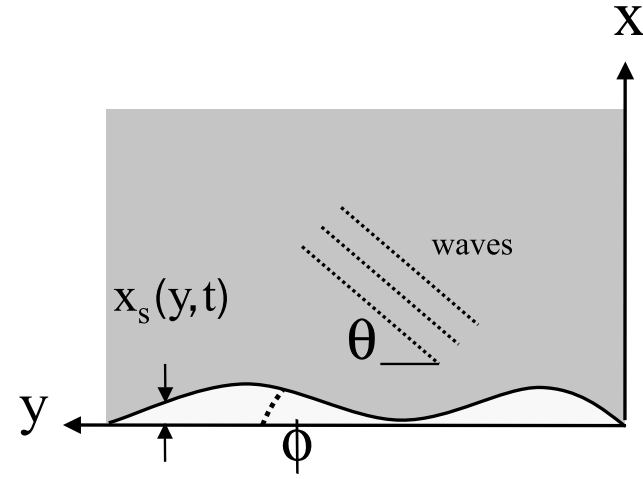

Figure 1. Sketch of the geometry and the variables. The angle between the wave fronts and the local shoreline is $\alpha=$ $\theta-\phi$.

always positive while the coastline instability implies a negative diffusivity. Falqués [2003] pointed out that the discrepancy lies on the fact that the traditional one-line model neglects the changes in wave height and angle at breaking caused by the changes in coastline orientation. This was found to be the cause of a significant overprediction of coastline diffusivity. However, both Ashton et al. [2001] and Falqués [2003] use two important simplifications: (1) any change of the coastline has its counterpart into the bathymetric lines up to deep water and (2) the modified bathymetric lines are rectilinear and parallel.

[5] The purpose of the present contribution is to take further the analysis of coastline instability and diffusivity initiated by those two papers by relaxing these hypotheses. In nature, the changes in the coastline are certainly linked to changes in the nearshore bathymetry but these changes extend only up to a finite distance from the shore. Furthermore, the departure from the initially rectilinear coast is an undulated shoreline so that the associated depth contours are not rectilinear and parallel. However, for morphological features with a very large alongshore length scale the curvature of the bathymetric lines will be very small so that the waves will feel almost rectilinear depth contours. In addition, it is relatively reasonable in this case to assume that the topographic perturbation may extend rather far offshore. Therefore it is expected that the results of both papers will hold for very large features. The main aim was to check whether that expectation is true and to which extend. However, by relaxing the rectilinear and parallel depth contours assumption and by introducing a finite offshore extend of the topographic perturbation new and interesting phenomena that were not foreseen in those two papers are now found. Formally, the procedure is to make a linear stability analysis of the rectilinear coastline with respect to small amplitude alongshore undulations. The complex growth rates provide the coastline diffusivity (in case of stability), a description of the high-angle waves instability (HAWI) and the alongshore propagation of shoreline sand waves.

[6] The new model is described in section 2 but some details of the perturbed wave transformation are left for Appendix A. The Assumptions on the reference topography of the rectilinear coast and on the perturbed bathymetry are explained in section 3 . The overall results regarding coast- line diffusivity and instability are presented in section 4 . Section 5 is devoted to the dominant wavelength for highangle wave instability. The physics underlying the model results is discussed in section 6. An application of the model to the Dutch coast is presented in section 7. Finally, an overview, concluding remarks and discussion of limitations of the model is presented in section 8 .

\section{Extended One-Line Formulation}

\subsection{Governing Equations}

[7] A cartesian coordinate system is assumed, $x$ seaward in the cross-shore direction, $y$ running alongshore and $z$ upward along the vertical. The aim is a description of the dynamics of the small departures of the coastline with respect to its rectilinear trend given by the $y$ axis. We will here extend the traditional one-line formulation according to which the dynamics of the coastline is described by the sediment conservation:

$$
\bar{D} \frac{\partial x_{s}}{\partial t}=-\frac{\partial Q}{\partial y},
$$

where $Q$ is the total sediment transport rate $\left(\mathrm{m}^{3} \mathrm{~s}^{-1}\right)$ in the $y$ direction, $t$ is time and the perturbed coastline is given by $x=x_{s}(y, t)$ [Pelnard-Considère, 1956; Horikawa, 1988; Komar, 1998]. The average active water depth $\bar{D}$ is of the order of the depth of closure, $D_{c}$, and a precise definition of it will be given in section 2.2. We will consider here the widely used CERC formula [Komar, 1998; Horikawa, 1988] for the sediment transport rate:

$$
Q=\mu H_{b}^{5 / 2}\left(\sin \left(2 \alpha_{b}\right)-\frac{2 r}{\beta} \cos \left(\alpha_{b}\right) \frac{\partial H_{b}}{\partial y}\right),
$$

where $H_{b}$ is the (rms) wave height and $\alpha_{b}$ is the angle between wave fronts and coastline at breaking (see Figure 1). The constant in front of it is of order $\mu \sim 0.1-$ $0.2 \mathrm{~m}^{1 / 2} \mathrm{~s}^{-1}$ and $\beta$ is the beach slope at the shoreline. According to Horikawa [1988] the nondimensional constant $r$ ranges between 0.5 and 1.5. The value $r=1$ has been used for our modeling. Other expressions could be worked out but as long as the qualitative trends with respect to $H_{b}$ and $\alpha_{b}$ are similar no essential differences are expected in the results.

[8] The computation of sediment transport with equation (2) requires the previous knowledge of $H_{b}(y, t)$ and $\alpha_{b}(y, t)$. These two quantities must be computed performing wave transformation from deep water and at this point the essential differences between the various approaches arise. The traditional formulation set up by Pelnard-Considere [1956] just ignores the perturbation in wave transformation caused by the coastline changes. This is equivalent to keep the bathymetric contours being parallel to the unperturbed rectilinear coastline even if changes in coastline position occur. The formulation used by Ashton et al. [2001] and Falqués [2003] assumes that if the coastline orientation changes the bathymetric contours change too, becoming parallel to the new coastline. There are however two important simplifications: the bathymetric changes extend up to deep water and wave transformation is made by assuming parallel depth contours which is inconsistent with 
the wavy shape of the perturbed coastline. The present model addresses these two limitations by considering a finite offshore extend of the bathymetric perturbations and by performing wave transformation on curvilinear depth contours. How the new model deals with wave transformation up to breaking is described below.

[9] The wave direction is measured by the angle between the wave rays and the $-x$ axis, represented by $-\theta$ and defined by

$$
k_{x}=-k \cos \theta(x, y) \quad k_{y}=k \sin \theta(x, y),
$$

where $\mathbf{k}$ is the wave number vector (see Figure 1). If the angle between the coastline and the $y$ axis is $\arctan \left(\partial x_{s} / \partial y\right)=\phi$, the angle between wave fronts and bathymetric contours at breaking will be $-\alpha_{b}=-\theta_{b}(y, t)+$ $\phi(y, t)$. Wave transformation from deep water is done by solving the wave front conservation and energy conservation equations

$$
\begin{gathered}
\nabla \times \mathbf{k}=0 \\
\nabla \cdot\left(H^{2} \mathbf{c}_{g}\right)=0
\end{gathered}
$$

together with the dispersion relation [Mei, 1989; Horikawa, 1988]

$$
\omega^{2}=g k \tanh (k D)
$$

and boundary conditions in deep water, $x=x_{\infty}$,

$$
H\left(x_{\infty}, y, t\right)=H_{\infty}(y, t) \quad \theta\left(x_{\infty}, y, t\right)=\theta_{\infty}(y, t) .
$$

A single monochromatic wave train with $H=H_{r m s}$ and $T=$ $T_{p}$ is considered here. The group velocity and the frequency are $\mathbf{c}_{g}$ and $\omega, D$ is the water depth and $g$ is gravity. Thus the governing equations of the morphodynamic system are equations (1), (4), and (5), with the boundary conditions (6) and sediment flux, equation (2).

\subsection{Dynamics of Small Departures From a Rectilinear Coastline}

[10] We will now derive the equation governing the dynamics of small perturbations of the rectilinear coastline, $x=x_{s}(y, t)$. Formally, the procedure is a linear stability analysis. Thus a basic reference state corresponding to the rectilinear coastline must be first found. Given an alongshore uniform topography $D=D_{0}(x)$ with $D_{0}(0)=0$, and a steady and uniform deep water wave input, $H_{\infty}(y, t)=$ const., $\theta_{\infty}(y, t)=$ const., a steady and alongshore uniform solution of our system is immediately found. The wave number $k_{0}(x)$ is readily computed from equation (5). The alongshore uniformity allows to cast equation (4a) into the Snell law which, together with equation (4b), read

$$
\frac{d}{d x}\left(k_{0} \sin \left(\theta_{0}\right)\right)=0 \quad \frac{d}{d x}\left(c_{g} \cos \left(\theta_{0}\right) H_{0}^{2}\right)=0,
$$

from where the wave incidence angle, $\theta_{0}(x)$, and wave height, $H_{0}(x)$, are found. Since $H_{b}$ and $\alpha_{b}$ are alongshore uniform, so is the sediment transport $Q$ and the steady and rectilinear coastline $x_{s}(y, t)=0$ is a solution of equation (1). Therefore

$$
x_{s}=0, \quad k=k_{0}(x), \quad \theta=\theta_{0}(x), \quad H=H_{0}(x)
$$

is a solution of the system and will hereinafter be considered as the basic reference state.

[11] We now consider a small deviation of the rectilinear coastline given by $x_{s}(y, t)$. Associated to it we will assume a topographic perturbation with total water depth given by

$$
D(x, y, t)=D_{0}(x)-h(x, y, t)=D_{0}(x)-\beta f(x) x_{s}(y, t),
$$

with a shape function $f(x)$ that verifies $f(0)=1$ and $f\left(x_{c}\right)=0$, where $x_{c}$ is the offshore distance where the depth of closure is reached, $D_{0}\left(x_{c}\right)=D_{c}$. The assumption of a fixed crossshore shape of the bathymetric perturbation is one of the important assumptions of the model and is fully consistent with the one-line coastline modeling concept. However, the present extension can be seen as 1.5-D since the 1-D morphodynamics induces bathymetric changes in $2 \mathrm{DH}$ that feedback into the waves which, in turn, govern coastline changes.

[12] Now, the one-line concept that all the sediment deficit or excess on a cross-shore section is spent on shoreline changes,

$$
\int_{0}^{x_{c}} h(x, y, t) d x=\bar{D} x_{s}(y, t)
$$

provides, by comparing with equation (9), a definition of the average active water depth:

$$
\bar{D}=\beta \int_{0}^{x_{c}} f(x) d x .
$$

Notice that this makes our formulation consistent regarding sediment conservation in $2 \mathrm{DH}$.

[13] The departure from rectilinear and parallel depth contours defined by equation (9) induces a perturbation on the wave transformation:

$$
\begin{aligned}
k= & k_{0}(x)+k^{\prime}(x, y, t), \quad \theta=\theta_{0}(x)+\theta^{\prime}(x, y, t), \quad H=H_{0}(x) \\
& +H^{\prime}(x, y, t),
\end{aligned}
$$

which is calculated in Appendix A. Once the perturbed wave transformation is known, the quantities defined at the breaking line which are needed to compute sediment transport can be evaluated. The position of the breaker line in the basic state is given by $H_{0}\left(x_{b}^{0}\right)=\gamma_{b} D_{0}\left(x_{b}^{0}\right)$. The same expression applied to the perturbed water depth and wave height at the perturbed breaker line,

$$
H\left(x_{b}^{0}+x_{b}^{\prime}\right)=\gamma_{b} D\left(x_{b}^{0}+x_{b}^{\prime}\right),
$$

allows to determine its position from

$$
x_{b}^{\prime}=\frac{H^{\prime}\left(x_{b}^{0}, y, t\right)+\gamma_{b} h\left(x_{b}^{0}, y, t\right)}{\gamma_{b} \frac{d D_{0}}{d x}\left(x_{b}^{0}\right)-\frac{d H_{0}}{d x}\left(x_{b}^{0}\right)} .
$$


The perturbations in wave angle and wave height at the perturbed breaking line,

$$
\begin{aligned}
& \theta_{b}^{\prime}(y, t)=\theta\left(x_{b}^{0}+x_{b}^{\prime}, y, t\right)-\theta_{0}\left(x_{b}^{0}\right) \quad H_{b}^{\prime}(y, t)=H\left(x_{b}^{0}+x_{b}^{\prime}, y, t\right) \\
& \quad-H_{0}\left(x_{b}^{0}\right),
\end{aligned}
$$

are then computed as

$\theta_{b}^{\prime}=\theta^{\prime}\left(x_{b}^{0}, y, t\right)+\frac{d \theta_{0}}{d x}\left(x_{b}^{0}\right) x_{b}^{\prime} \quad H_{b}^{\prime}=H^{\prime}\left(x_{b}^{0}, y, t\right)+\frac{d H_{0}}{d x}\left(x_{b}^{0}\right) x_{b}^{\prime}$.

[14] Finally, owing to the linearized relationship

$$
\alpha_{b}=\theta_{b}-\phi \simeq \theta_{b}-\frac{\partial x_{s}}{\partial y}
$$

the morphodynamic governing equation

$$
\begin{aligned}
\frac{\partial x_{s}}{\partial t}= & \frac{2 \mu\left(H_{b}^{0}\right)^{5 / 2}}{\bar{D}}\left(\left(\frac{\partial^{2} x_{s}}{\partial y^{2}}-\frac{\partial \theta_{b}^{\prime}}{\partial y}\right) \cos \left(2 \theta_{b}^{0}\right)-\frac{5}{4 H_{b}^{0}} \frac{\partial H_{b}^{\prime}}{\partial y} \sin \left(2 \theta_{b}^{0}\right)\right. \\
& \left.+\frac{r}{\beta} \cos \left(\theta_{b}^{0}\right) \frac{\partial^{2} H_{b}^{\prime}}{\partial y^{2}}\right)
\end{aligned}
$$

readily follows from the linearized equations (1) and (2). It is immediately seen that this equation reduces to the classical one-line equation

$$
\frac{\partial x_{s}}{\partial t}=\epsilon_{\mathrm{cla}} \frac{\partial^{2} x_{s}}{\partial y^{2}}
$$

with

$$
\epsilon_{\text {cla }}=\frac{2 \mu}{\bar{D}}\left(H_{b}^{0}\right)^{5 / 2} \cos \left(2 \theta_{b}^{0}\right)
$$

if the perturbations in wave angle and wave height are neglected [Falqués, 2003]: $\theta_{b}^{\prime}=0, H_{b}^{\prime}=0$. Notice that the governing equation, in contrast with equation (19), is non local. This means that $\partial x_{s}(t) / \partial t$ at a particular location $y=y_{1}$ can not be determined only from $x_{s}(t)$ and its $y$ derivatives at $y=y_{1}$. This is because $\partial x_{s}(y, t) / \partial t$ depends on the evaluation of $\theta_{b}^{\prime}, H_{b}^{\prime}$ and the latter quantities depend on the integration of $\theta^{\prime}(x, y, t), H^{\prime}(x, y, t)$ in all the nearshore domain which in turn depends on the values of $x_{s}(y, t)$ for all $y$ through the perturbed bathymetry.

[15] The difficulty of a nonlocal governing equation can be easily overcome by considering that any initial perturbation can be expanded in Fourier modes so that the behavior of the individual wave-like disturbances permits to reconstruct the dynamics of arbitrary perturbations because of the linearity of equation (18). Therefore we will hereinafter consider perturbations of the form

$$
x_{s}(y, t)=a e^{\sigma t+i m y}+\text { c.c. },
$$

with the associated perturbation in the bathymetry

$$
D=D_{0}(x)-\hat{h}(x) e^{\sigma t+i m y}+\text { c.c. },
$$

where the bathymetric perturbation is given by $\hat{h}(x)=\beta a f(x)$. Similar expressions are assumed for the wave quantities (see Appendix A). By inserting these expressions in equation (18) the growth rate $\sigma$ can be computed as

$$
\begin{aligned}
\sigma= & -2 m \frac{\mu}{\bar{D}}\left(H_{b}^{0}\right)^{5 / 2}\left\{\left(m+i \frac{\hat{\theta}_{b}^{\prime}}{a}\right) \cos \left(2 \theta_{b}^{0}\right)\right. \\
& \left.+\left(\frac{r m}{\beta} \cos \left(\theta_{b}^{0}\right)+\frac{5 i}{4 H_{b}^{0}} \sin \left(2 \theta_{b}^{0}\right)\right) \frac{\hat{H}_{b}^{\prime}}{a}\right\} .
\end{aligned}
$$

The imaginary part of the growth rate, $\sigma_{i}$, provides the alongshore celerity of each wave-like disturbance:

$$
V(m)=-\frac{\sigma_{i}(m)}{m}
$$

The sign of the real part of the growth rate, $\sigma_{r}$, indicates whether the perturbation will grow (instability) or decay (stability). In case of stability, by comparison with equation (19), the "diffusivity" felt by each wave-like solution will be

$$
\epsilon(m)=-\frac{\sigma_{r}(m)}{m^{2}}
$$

Notice that even though the governing equation is not an advection-diffusion one, a wavelength-dependent celerity and diffusivity can still be defined by equations (24) and (25).

\section{Coastal Topography}

[16] In order to proceed further with an investigation of coastline diffusivity and HAWI, it is necessary to prescribe certain topography in the basic state and certain shape function for the topographic perturbation.

\subsection{Equilibrium Topography}

[17] The equilibrium beach profile is taken as a Dean profile type:

$$
D_{0}(x)=b\left(\left(x+x_{0}\right)^{2 / 3}-x_{0}^{2 / 3}\right)
$$

which has been modified to avoid an infinite slope at the shoreline. The constants $b$ and $x_{0}$ are determined in order to have a prescribed slope $\beta$ at the coastline and a prescribed distance $x_{c}$ from the coastline to the location of the depth of closure, $D_{c}$. Thus $b$ and $x_{0}$ are solution of

$$
\frac{2}{3} b x_{0}^{-1 / 3}=\beta \quad b\left(\left(x_{c}+x_{0}\right)^{2 / 3}-x_{0}^{2 / 3}\right)=D_{c} .
$$

\subsection{Submerged Topography of the Sand Wave}

[18] Regarding the topographic perturbation associated to the sand wave, different choices are possible, specially in account of the lack of detailed observations of the submerged part of the sand waves. Looking at the short-term dynamics (hours to days) an excess or deficit of sand in the surf zone can trigger a complex dynamics involving sand bars and rhythmic topography. However, in the long term (months to years) it is expected that the cross-shore beach 
profile will tend to some sort of equilibrium profile. Thus a sand wave crest or horn would be associated to an offshore shift of the equilibrium profile at the corresponding crossshore section while a bay will be associated to an onshore shift of the profile. This assumption is strictly consistent with the traditional one-line modeling concept and would imply a bathymetric perturbation with a shape function

$$
f(x)=\frac{1}{\beta} \frac{d D_{0}(x)}{d x} .
$$

This perturbation decreases offshore as does the slope in the equilibrium profile but it extends up to deep water and would imply bathymetric changes beyond the depth of closure, $D_{c}$, which is not realistic. On the other hand, the equilibrium profile concept is based on alongshore uniformity and steadiness while the sand wave is in fact dynamic and alongshore non uniform by definition. These two facts make the strict application of the shift of the equilibrium profile, equation (28), unadvisable. Thus we will use instead the bathymetric perturbation given by

$$
f(x)=\frac{e^{-x / L}-e^{-x_{c} / L}}{1-e^{-x_{c} / L}}
$$

which vanishes at the depth of closure and where $L$ controls the offshore decay of the bathymetric disturbance. A small $L$ defines a bathymetric perturbation confined next to the coast while large values are associated with a perturbation decaying almost linearly from the coastline up to the depth of closure, $x_{c}$. There is freedom in the model to choose any value of $L$ and its influence on the diffusivity and the highangle wave instability has been investigated. However, if we admit that there is a tendency for the cross-shore profile to adjust to the shifted equilibrium profile, $L$ should not be far away from the value that causes the curves given by equations (28) and (29) to match close to the coast. This gives an indication of the realistic range of that parameter.

\section{Coastline Diffusivity and Instability}

\subsection{Scaling and Parameter Setting}

[19] In addition to the three nondimensional parameters $\theta_{\infty}, \beta$ and $\gamma_{b}$, the model has seven independent parameters which are lengths. These are first the offshore distance where deep water wave characteristics are given, $x_{\infty}$, and the wave height and wavelength at that position, $H_{\infty}, 2 \pi /$ $k_{\infty}$. Furthermore, there is the depth of closure, $D_{c}$, at a distance $x_{c}$ from the shore. Finally, we should mention the $L$ distance and the wavelength of the sand waves, $\lambda$.

[20] Since the wave period can be related to $k_{\infty}$ through $g$, there is only one independent timescale which is related to the morphodynamics. It is given by the coefficient $\mu$ in the sediment transport rate and, in view of equation (23), a natural choice for it is

$$
T_{m}=\frac{\bar{D}}{\mu m^{2}}\left(H_{b}^{0}\right)^{-5 / 2} .
$$

[21] Thus because of the scale invariance, if all the distances are multiplied by a certain factor $\rho$, everything

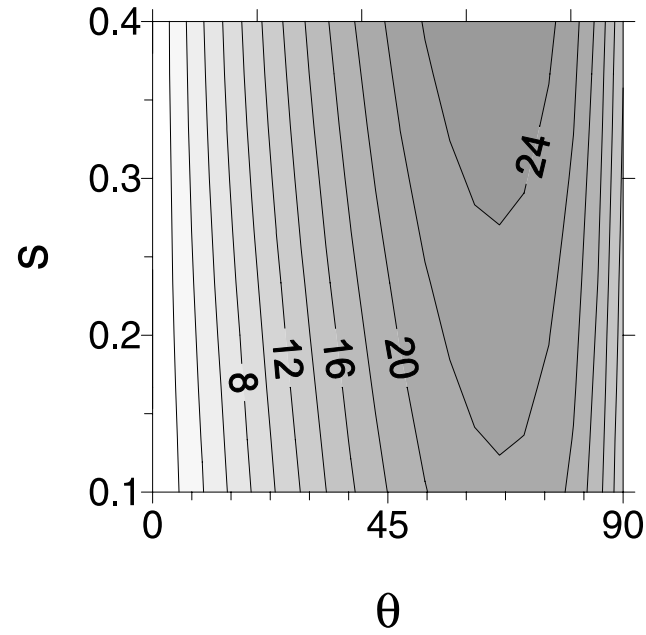

Figure 2. Wave angle at breaking, $\theta_{b}$, as a function of the angle, $\theta_{\infty}$, and wave steepness parameter, $s=k_{\infty} H_{\infty}$, given at $x_{\infty}=2 \times 10^{5} \mathrm{~m}$, with $D_{\infty}=57 \mathrm{~m}$.

remains unchanged but with a timescale multiplied by $\rho^{1 / 2}$. It is therefore convenient to use a basic length scale and keep it unchanged for the numerical experiments. It has been found that the best choice is the depth of closure, $D_{c}$. This allows defining six nondimensional parameters as the ratios of the other six distances to $D_{c}$. However, based on the physics and/or practical reasons, some combinations of them will be sometimes used instead. For instance, the main ratio of lengths for the model runs is $s=k_{\infty} H_{\infty}$ which is a measure of the wave steepness in deep water. It is an indicator of the type of waves, small values corresponding to swell and large values to sea. It comes into play because it is roughly proportional to $k_{b} D_{b}$ so that it is a measure of the strength of the topographic effects on the waves propagating from deep water up to breaking. Although we run the model giving the value of $H_{\infty}$, the corresponding relevant nondimensional parameter is $D_{b} / D_{c}$ which is roughly proportional to $H_{\infty} / D_{c}$ and gives the width of the surf zone relative to $x_{c}$. Since for fixed $H_{\infty}$ it depends on the angle, the values used in the text are always values for $\theta_{\infty}=$ 0 . The rest of nondimensional parameters will be defined as necessary in the text.

[22] The depth of closure has been fixed to $D_{c}=10 \mathrm{~m}$ and the outer boundary where deep water wave characteristics are prescribed has been fixed to $x_{\infty}=2 \times 10^{4} \mathrm{~m}$. Small changes around the latter value did not have any significant change on the results. The breaking index has been set to $\gamma_{b}=0.8$. The diffusivity has been scaled with its traditional evaluation, $\epsilon_{\text {cla }}$, given by equation (20). Several values of $s$ have been investigated but we will show results only for $s=0.03,0.2$ and 0.4 ranging from swell to sea. For a wave height in deep water of $H_{\infty}=1 \mathrm{~m}$, the corresponding wave periods were $T=13,4.5$ and 3.2 s., respectively. The wave incidence angle ranged from $\theta_{\infty}=0^{\circ}$ to $89^{\circ}$ and the wavelength of the coastline waves has been explored from $\lambda=1000 \mathrm{~m}$ up to $2 \times 10^{4} \mathrm{~m}$. The default values of the topographic parameters have been chosen as roughly representative of the Dutch coast, $\beta=0.01$ and the depth of closure of $D_{c}=10 \mathrm{~m}$ occurring at an offshore 

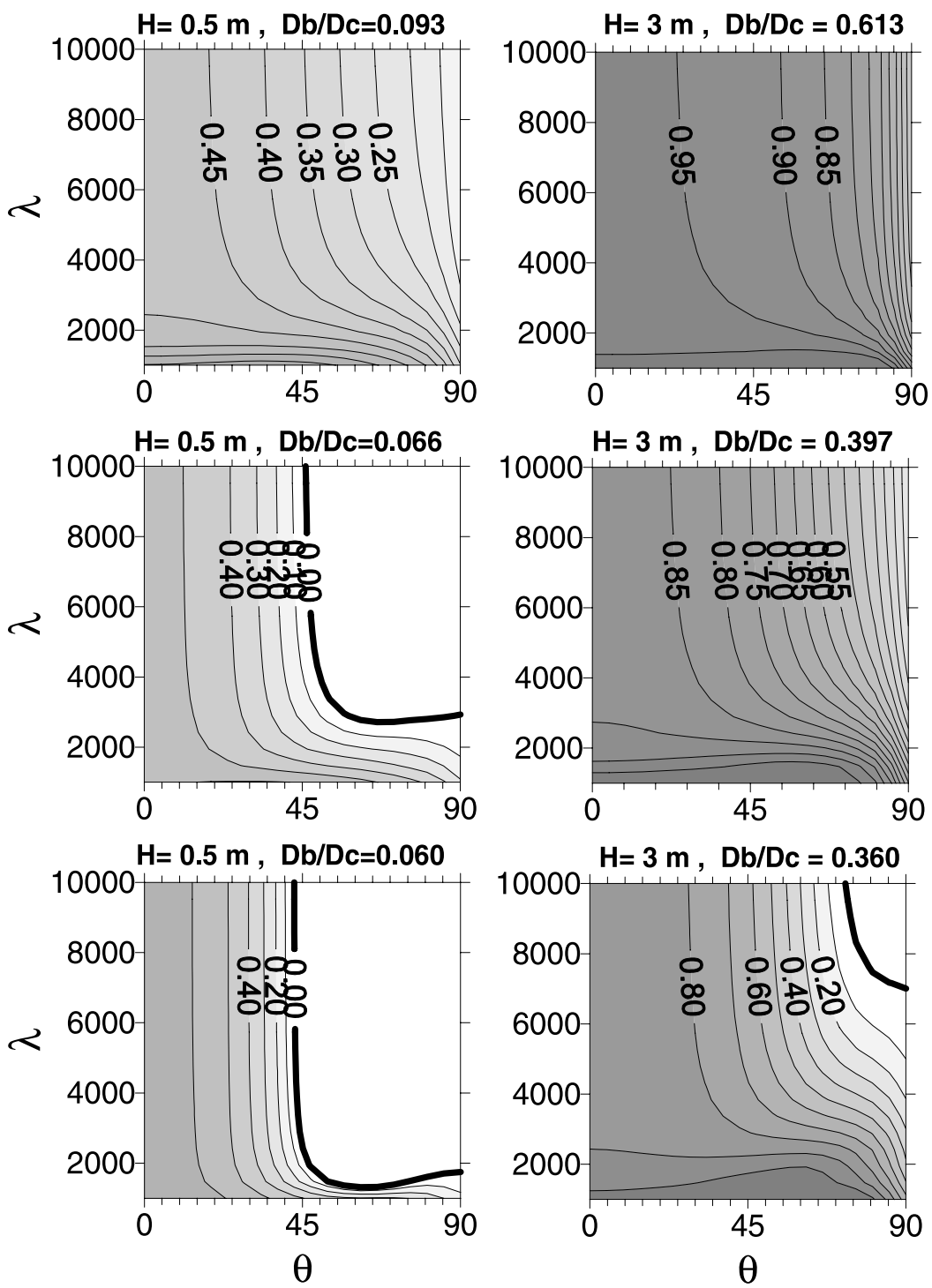

Figure 3. Ratio between the diffusivity and its classical evaluation, $\epsilon / \epsilon_{\text {cla }}$, as a function of wave incidence angle in deep water, $\theta_{\infty}$, and the wavelength of the coastline feature, $\lambda$, for various wave steepnesses $s$ and for various wave heights in deep water, $H_{\infty}$. (top) Corresponding to $s=0.03$. (middle) Corresponding to $s=0.2$. (bottom) Corresponding to $s=0.4$. (left) Corresponding to $H_{\infty}=0.5 \mathrm{~m}$. (right) Corresponding to $H_{\infty}=3 \mathrm{~m}$. The thick line is the zero-diffusivity contour line, indicating the threshold for the high-angle waves instability (HAWI).

distance $x_{c}=1750 \mathrm{~m}$. An $L=700 \mathrm{~m}$ value is used unless stated otherwise.

\subsection{General Results}

[23] In a first series of experiments, computations have been carried out in order to investigate the diffusivity affecting the various wave-like perturbations as a function of wavelength $\lambda=2 \pi / m$, wave incidence angle $\theta_{\infty}$, wave steepness $s$ and $D_{b} / D_{c}$ parameter. The influence of the wave incidence angle at breaking, $\theta_{b}$, can be tracked through the dependence on $\theta_{\infty}$ by using Figure 2 where $\theta_{b}$ is shown as a function of $\theta_{\infty}$ and $s$. In agreement with Falqués [2003], it is found that the scaled diffusivity, $\epsilon / \epsilon_{\text {cla }}$, is typically smaller than 1 which means that the traditional evaluation of the diffusivity systematically overpredicts it. In general terms, the scaled diffusivity increases with increasing $D_{b} / D_{c}$ and with decreasing wave steepness $s$ and wave angle $\theta_{\infty}$. The diffusivity does not depend on wavelength for long waves but it increases for decreasing wavelength within the short wave range $(\lambda \sim 2-4 \mathrm{~km})$ and, occasionally, it can even be slightly larger than 1 . As it can be seen in Figure 3, for large waves, i.e., large $D_{b} / D_{c}$, the ratio is rather close to 1 , specially for swell and for moderate incidence angle. For instance, for $D_{b} / D_{c}=0.613$ and moderate angle, $\epsilon / \epsilon_{\mathrm{cla}}$ is about $0.8-1$. Thus for large swell waves the classical diffusivity evaluation is rather good and there is no HAWI. This can be seen, for instance, in case of $s=0.03$ where the diffusivity is always positive. Computations not shown here, indicate that for very high waves, for instance, $H_{\infty}=$ $5 \mathrm{~m}$ and $s=0.1\left(D_{b} / D_{c}=0.826\right)$, there is no HAWI and the classical prediction of the diffusivity is very good even for quite oblique wave incidence (errors smaller than $5 \%$ for $\theta_{\infty}$ 


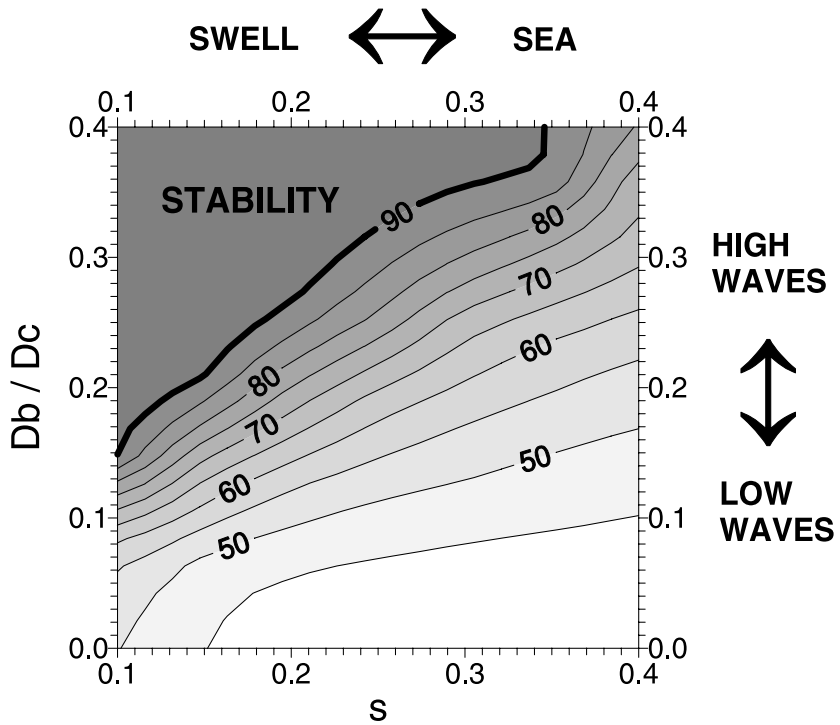

Figure 4. Critical angle in deep water for HAWI as a function of wave steepness $s$ and $D_{b} / D_{c}$.

up to $60^{\circ}$ ). This is specially so for relatively large wave periods.

[24] By decreasing wave height the ratio decreases. This is more prominent for sea waves than for swell and the decrease becomes more pronounced for large incidence angle. For small $D_{b} / D_{c}$ the ratio becomes more dependent on $\lambda$, specially for relatively short features, $\lambda \sim 2000$ $4000 \mathrm{~m}$. In this range, i.e., quite oblique and relatively small sea waves, the picture becomes more complex and the classical evaluation of the diffusivity is no longer valid. As it can be seen in Figure 3, the overprediction by the classical formulation can easily be a factor 10 since it increases without bound by approaching the HAWI threshold. Whether HAWI occurs or not depends on $\theta_{\infty}, s$ and $D_{b} / D_{c}$. This can be seen in Figure 3 and also in Figure 4, where the critical angle for the onset of instability is plotted as a function of $s$ and $D_{b} / D_{c}$. Interestingly, the critical angle for the most unstable case is about $42^{\circ}$, the one predicted by Ashton et al. [2001]. However, turning to more stable situations with higher waves and longer periods, the critical angle increases and at some point there is no longer HAWI for any angle.

[25] For the sake of brevity the influence of changing $L$ is not discussed in detail here. It turns out that decreasing (increasing) $L$ is somewhat similar to increasing (decreasing) $D_{b} / D_{c}$. For small $L$, that is for a bathymetric perturbation hardly extending offshore, the ratio $\epsilon / \epsilon_{\text {cla }}$ is close to 1 and there is no HAWI. By increasing $L$, that ratio decreases and the coastline may become unstable. Further increase up to values comparable to $x_{c}$ do not have any effect since the shape function $f(x)$ tend to a straight line for $L \rightarrow \infty$.

[26] The present results can also be compared to the extension of the classical approach by Falqués [2003] where the dependence of $\theta_{b}$ and $H_{b}$ on $\phi$ was accounted for. In principle, it seems that the present model and that extension should coincide for large $\lambda$ and $L$. However, this is the case only for sea waves. In case of swell, the earlier results reproduce only the trend with the angle but not the magnitude of $\epsilon / \epsilon_{\text {cla }}$ for large $L$.

\subsection{Influence of the Equilibrium Topography}

[27] In order to asses the influence of the equilibrium topography, moderately steep sea waves with $s=0.2$ and with $H_{\infty}=1 \mathrm{~m}\left(D_{b} / D_{c}=0.13\right)$ were used. For each beach profile, $L$ was chosen so as to match the equilibrium profile
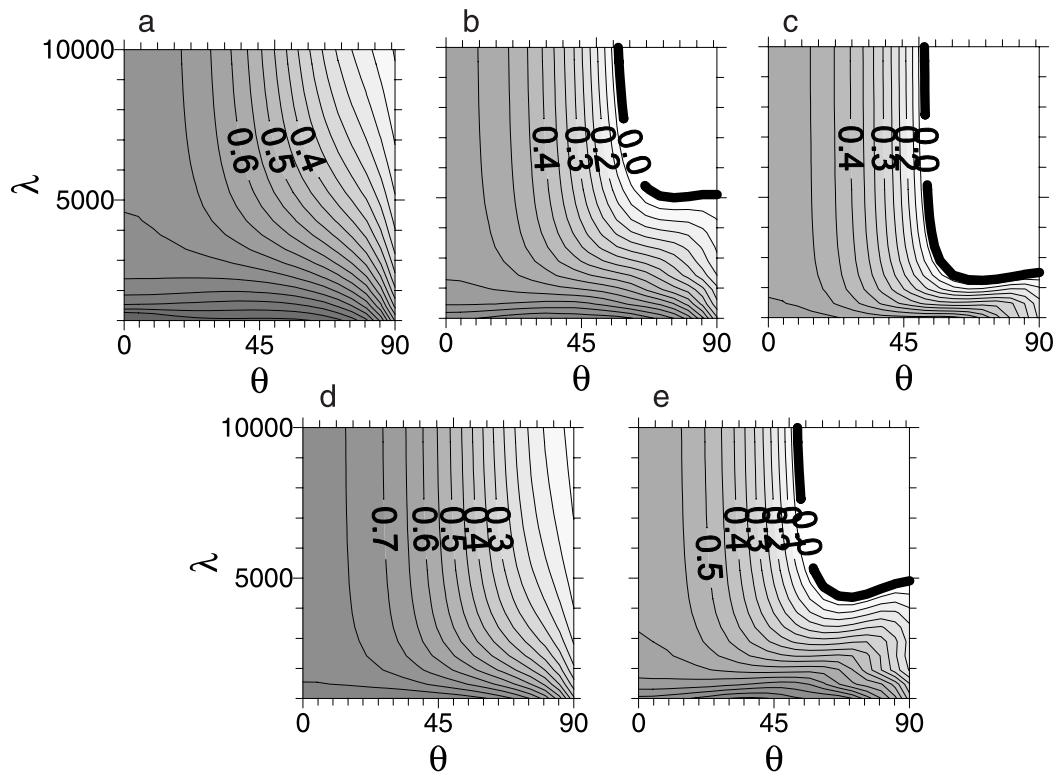

Figure 5. Ratio between the diffusivity and its classical evaluation, $\epsilon / \epsilon_{\text {cla }}$, as a function of wave incidence angle in deep water, $\theta_{\infty}$, and the wavelength of the coastline feature, $\lambda$, for a wave steepness $s=$ 0.2 and for various equilibrium topographies. The thick line is the zero-diffusivity contour line, indicating the threshold for HAWI. (a) $\beta=0.005, x_{c}=4498 \mathrm{~m}$, and $L=682 \mathrm{~m}$. (b) $\beta=0.01, x_{c}=1750 \mathrm{~m}$, and $L=$ $698 \mathrm{~m}$. (c) $\beta=0.02, x_{c}=716 \mathrm{~m}$, and $L=1084 \mathrm{~m}$. (d) $\beta=0.01, x_{c}=2390 \mathrm{~m}$, and $L=293 \mathrm{~m}$. (e) $\beta=0.01$, $x_{c}=1395 \mathrm{~m}$, and $L=3103 \mathrm{~m}$. 

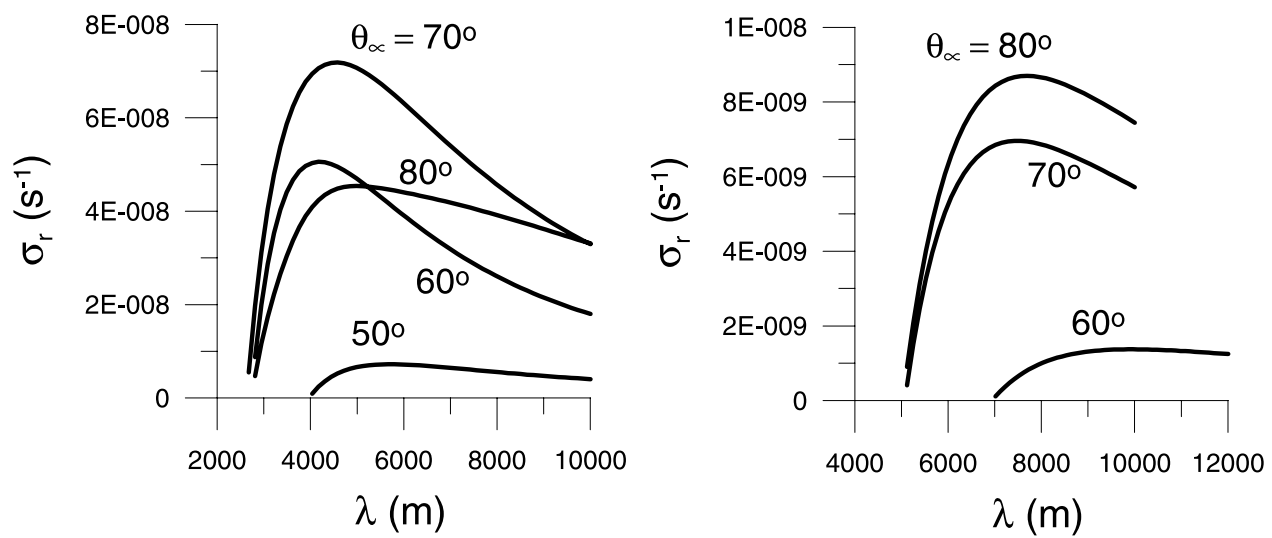

Figure 6. Instability curves (real growth rate versus wavelength) for $\beta=0.01$ and $x_{c}=1750 \mathrm{~m}$. (left) $s=$ 0.4 . (right) $s=0.2$.

shift close to the coastline (see end of section 3.2). Three types of tests have been performed. In the first one, the equilibrium profile has been simply moved up or down, just by dividing or multiplying $D_{0}(x)$ by a factor 2 . This is done by considering the three cases: $\beta=0.01, x_{c}=1750 \mathrm{~m}, \beta=$ $0.005, x_{c}=4498 \mathrm{~m}$ and $\beta=0.02, x_{c}=716 \mathrm{~m}$. As it can be seen in Figure 5 (top), a shallow profile gives a higher coastline diffusivity and inhibits high-angle wave instabil-
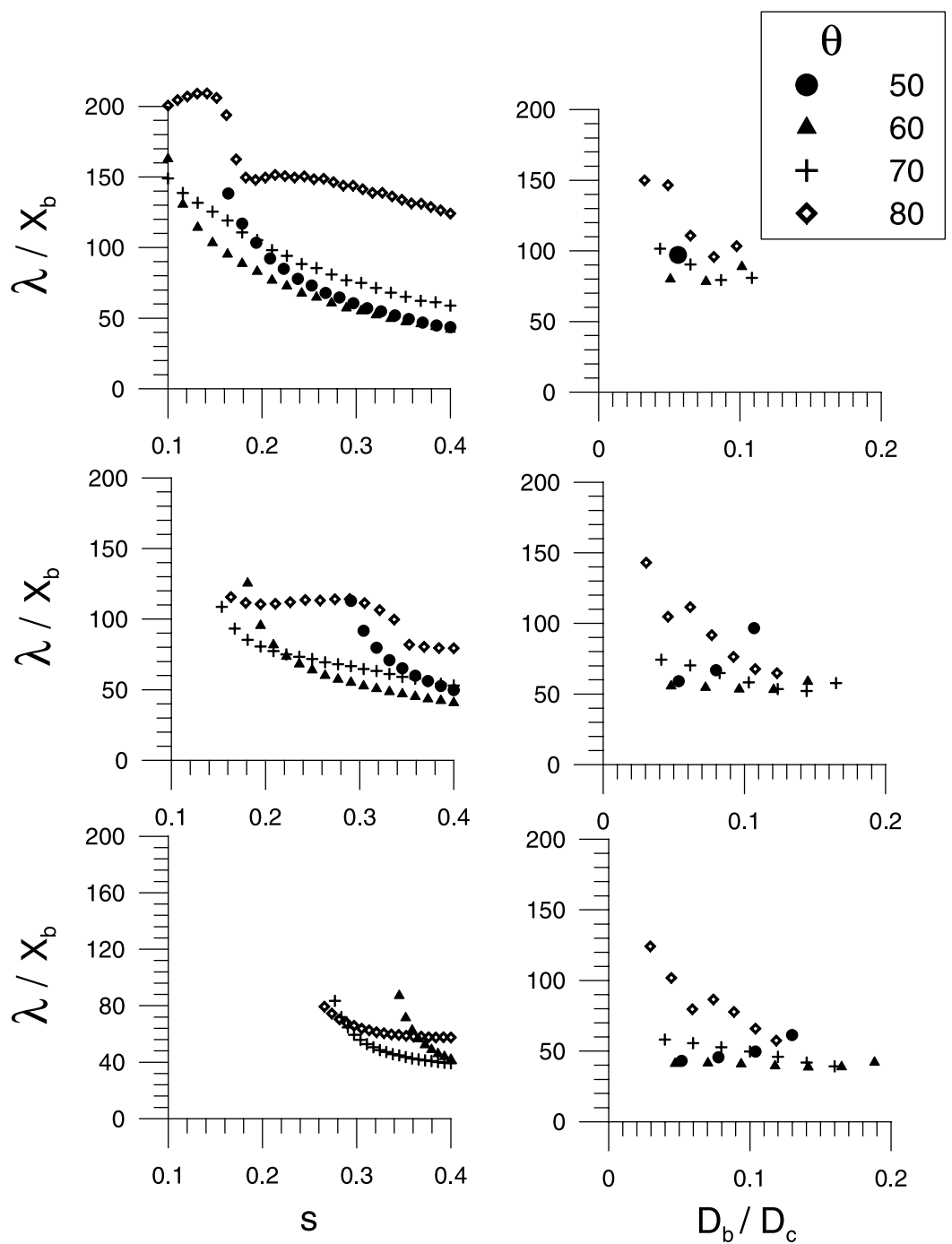

Figure 7. Ratio $\lambda_{M} / X_{b}$ as a function of (left) $s$ and as a function of (right) $D_{b} / D_{c}$ for various wave angles, $\theta_{\infty}=50^{\circ}, 60^{\circ}, 70^{\circ}$, and $80^{\circ}$, and for $\gamma_{b}=0.8$. The plots on the left are for $H_{\infty}=0.5,1$, and $2 \mathrm{~m}$, from top to bottom; the plots on the right are for $s=0.2,0.3$, and 0.4 , from top to bottom. 
ity. In the second case listed above there is no instability at all even for very oblique waves. In contrast, deeper profiles favor coastline instability.

[28] In the second type of experiments we looked at the effect of an offshore shallower or deeper profile while keeping the same slope at the shoreline. Thus $\beta=0.01$, $x_{c}=2390 \mathrm{~m}$ and $\beta=0.01, x_{c}=1395 \mathrm{~m}$ were considered. This is shown in Figure 5 (bottom). Again, the same effect is found, shallower profiles render the coastline more stable to high-angle waves. Finally, the shoreline slope was changed, $\beta=0.008$ or $\beta=0.012$, while keeping constant the offshore distance of the depth of closure, $x_{c}=1750 \mathrm{~m}$. In this case, there was no significant influence on the diffusivity and instability. In summary, it seems that the diffusivity and instability of the coastline is very sensitive to the water depth at the shoreface but not too much to the shoreline slope. For a given wave height and wave period, coastline diffusivity is smaller on deeper than on shallower shorefaces so that deeper shorefaces are more conducive to coastline instability.

\section{Dominant Wavelength for High-Angle Wave Instability}

[29] It has been shown in section 4 that the diffusivity may become negative for certain parameter values (see Figures 3 and 5) rending the rectilinear coastline unstable. We will focus in this section on the interesting finding that the instability depends on the wavelength of the coastline undulations, $\lambda$. As it can be seen in those figures, for any angle leading to instability the diffusivity becomes positive if the wavelength is decreased enough. Thus small coastline undulations do not grow. This, in turn, has the important consequence that for each parameter setting the instability has a preferred (dominant) wavelength in the linear regime as it can be seen in Figure 6. This is an essential characteristic of high-angle instability not predicted by the simplified model of Ashton et al. [2001]. Figure 6 also shows the typical magnitude of the real growth rate of the instability which turn to be in the order $10^{-8} \mathrm{~s}^{-1}$. In other words, the typical growth times are in the order of $1-10$ years. This long timescale is consistent with the whole long-term modeling concept of the present paper.

[30] The dominant wavelength for HAWI is typically in the range of $4-15 \mathrm{~km}$, that is, one to two orders of magnitude larger than the typical wavelength of rhythmic surf zone bars. In order to investigate its relationship with the fundamental length scales of the problem we first considered a plane sloping coast instead of the Dean-type profile defined in section 3.1. This was useful in a first instance to exclude parameter dependencies that could mask the main trends. In this case, by changing $H_{\infty}$ and $\beta$, the dominant wavelength $\lambda_{M}$ was fairly proportional to the width of the surf zone, $X_{b}$. This suggested that the ratio $\lambda_{M} / X_{b}$ is a good parameter to investigate also in case of more realistic bathymetries like the Dean-type profile.

[31] In case of non planar topography the wave height $H_{\infty}$ also plays an important role and the relevant nondimensional parameter is $D_{b} / D_{c}$. This is seen for the Deantype profile in Figure 7 (left panels) where $\lambda_{M} / X_{b}$ is shown as a function of wave steepness for $H_{\infty}=0.5,1$ and $2 \mathrm{~m}$. It turns out that $\lambda_{M} / X_{b}$ typically ranges between 40 and 150 and it is seen that it tends to increase for decreasing wave height and for decreasing $s$. Notice that $s / \gamma_{b}=2 \pi H_{\infty} /\left(\lambda_{w} \gamma_{b}\right)$ is related to $D_{b} / \lambda_{w}$ and hence gives an indication of the strength of the topographic effects on the waves of wavelength $\lambda_{w}$ propagating from deep water up to breaking. This also implies that the sand wave wavelength increases with increasing water wave wavelength. The right panels display $\lambda_{M} / X_{b}$ against $D_{b} / D_{c}$ for $s=0.2,0.3$ and 0.4. Similar trends are observed: an increase of $\lambda_{M} / X_{b}$ for decreasing $D_{b} / D_{c}$ and for decreasing $s$. The ratio $\lambda_{M} / X_{b}$ tends to increase with increasing angle although this tendency is reversed for low angles close to the instability threshold $\theta_{\infty} \sim 50^{\circ}$.

[32] As a result of our numerical study it turns out that the dominant sand wave wavelength can be expressed as the following nondimensional relationship:

$$
\frac{\lambda_{M}}{X_{b}}=\Lambda\left(\theta_{\infty}, \frac{s}{\gamma_{b}}, \frac{D_{b}}{D_{c}}\right) .
$$

There is also some influence of $\beta_{c} / \beta$, where $\beta_{c}=D_{c} / x_{c}$ is the mean slope of the shoreface, when this parameter drops below $\beta_{c} / \beta \simeq 0.5$. In this case, $\lambda_{M} / X_{b}$ decreases with decreasing $\beta_{c} / \beta$.

\section{Physical Interpretation}

\subsection{High-Angle Wave Instability: Simple Approach}

[33] Both the traditional one-line approach and the formulation of Ashton et al. [2001] actually neglect the curvature of the coastline and handle the coastline undulations as small stretches of rectilinear coast with different orientations given by the angle $\phi=\arctan \left(\partial x_{s} / \partial y\right)$. Before proceeding with the new formulation we can gain some insight by looking again at that simple approach. By assuming rectilinear and parallel depth contours, $H_{b}^{\prime}$ and $\theta_{b}^{\prime}$ can be computed as a function of $\phi$. Then, by applying the chain rule to equation (18) and assuming small $\phi$, the following equation is readily obtained:

$$
\begin{aligned}
\frac{\partial x_{s}}{\partial t}= & 2 \mu \frac{\left(H_{b}^{0}\right)^{5 / 2}}{\bar{D}}\left(\left(1-\frac{\partial \theta_{b}^{\prime}}{\partial \phi}\right) \cos \left(2 \theta_{b}^{0}\right)-\frac{5}{4 H_{b}^{0}} \frac{\partial H_{b}^{\prime}}{\partial \phi} \sin \left(2 \theta_{b}^{0}\right)\right) \\
& \cdot \frac{\partial^{2} x_{s}}{\partial y^{2}}+2 \mu \cos \left(\theta_{b}^{0}\right) \frac{r\left(H_{b}^{0}\right)^{5 / 2}}{\beta \bar{D}} \frac{\partial H_{b}^{\prime}}{\partial \phi} \frac{\partial^{3} x_{s}}{\partial y^{3}}
\end{aligned}
$$

It is immediately seen that if the dependence of wave angle and wave height on the angle of the coastline, $\phi$, is neglected, this equation becomes the classical one equation (19) with a positive diffusivity unless $\theta_{b} \geq 45^{\circ}$. Owing to the reduction of wave angle by topographic refraction when approaching the shore, the wave angle at breaking is typically smaller than $45^{\circ}$ [see Falqués, 2003, Figures 1 and 2. Thus without those dependencies on $\phi$, HAWI would not be any relevant for the dynamics of natural coastlines. However, by rotating the coastline toward the wave fronts (i.e., by increasing $\phi$ ) the refraction is reduced (i.e., $\theta_{b}^{\prime}$ increases) and the wave height at breaking is increased (less wave energy spreading due to divergence of wave rays). Thus both $\partial \theta_{b}^{\prime} / \partial \phi$ and $\partial H_{b}^{\prime} / \partial \phi$ are positive with the result that the term within the brackets in equation (32) changes sign below $\theta_{b}=45^{\circ}$ and hence the high-angle instability occurs for realistic wave conditions as was first found by 
Ashton et al. [2001]. These dependencies are therefore essential for HAWI.

[34] The counterpart of the last statement in the new formulation follows by looking at the expression for the growth rate which derives from equation (23):

$$
\begin{aligned}
\sigma_{r}= & 2 m^{2} \frac{\mu}{\bar{D}}\left(H_{b}^{0}\right)^{5 / 2} \cos \left(2 \theta_{b}^{0}\right)\left(\frac{\hat{\theta}_{b i}^{\prime}}{a m}+\frac{5 \hat{H}_{b i}^{\prime}}{4 a m H_{b}^{0}} \tan \left(2 \theta_{b}^{0}\right)-1\right) \\
& -2 m^{2} \frac{\mu r}{\beta \bar{D}}\left(H_{b}^{0}\right)^{5 / 2} \cos \left(2 \theta_{b}^{0}\right) \frac{\hat{H}_{b r}^{\prime}}{a} .
\end{aligned}
$$

Since the maximum in wave energy is located closer to the crests of the sand wave than the bays, $H_{b r}^{\prime}>0$ and it becomes clear that instability $\left(\sigma_{r}>0\right)$ is associated to $\hat{\theta}_{b i}^{\prime}>0$ and $\hat{H}_{b i}^{\prime}>0$.

\subsection{Physics of the Instability}

[35] Although the discussion based on the analysis of the various terms in the growth rate expression, equation (33), already provides the link between hydrodynamics and morphodynamics, a simple discussion based on gradients in sediment transport as by Ashton et al. [2001] would be desirable at this point. However, if the bathymetric contours are not rectilinear and parallel, the sediment transport rate is no longer a function of the local shoreline orientation since it depends on wave transformation on the whole domain (and, in particular, on $\lambda$ ). Thus the simple analysis based on the curve $Q=Q\left(\theta_{b}-\phi\right)$, presented in that paper is not possible anymore. Yet rephrasing the discussion we already based on $\theta_{b}^{\prime}$ and $H_{b}^{\prime}$ now in terms of gradients of $Q$ is possible and enlightening.

[36] The perturbation of the sediment transport rate equation (2) gives

$$
\begin{aligned}
Q^{\prime}= & 2 \mu a H_{b}^{5 / 2} \cos 2 \theta_{b}^{0}\left(\frac{\theta_{b}^{\prime}}{a}+\frac{5 H_{b}^{\prime}}{4 a H_{b}^{0}} \tan 2 \theta_{b}^{0}-i m-i r m \frac{H_{b}^{\prime}}{\beta a} \frac{\cos \theta_{b}^{0}}{\cos 2 \theta_{b}^{0}}\right) \\
& =Q_{r}^{\prime}+i Q_{i}^{\prime},
\end{aligned}
$$

with the im term coming from the change in orientation of the coastline. The growth or decay and the possible migration of the sand waves is determined by the position of the maximum accretion points with respect to the adjacent crests and this maximum is located just $\lambda / 4$ downdrift of the maximum in $Q$. Then, the maximum in $Q$ is located at the crests in case of $Q_{r}^{\prime}>0, Q_{i}^{\prime}=0$ and $\lambda / 4$ updrift of the crests for $Q_{r}^{\prime}=0, Q_{i}^{\prime}>0$. In the general case, the four possible combinations of signs of $Q_{r}^{\prime}$ and $Q_{i}^{\prime}$ alone will determine in which quadrant of wavelength the maximum is located.

[37] Without perturbations in wave angle and wave height, $\theta_{b}^{\prime}=0, H_{b}^{\prime}=0$, the sediment transport rate is $Q_{r}^{\prime}=0, Q_{i}^{\prime}<0$ so that the maximum transport occurs at a distance $\lambda / 4$ downdrift of the horns and the maximum accretion occurs exactly at the bays. This is exactly what happens with the classical approach, any perturbation being eroded without alongshore migration. This situation changes when the other two terms are considered. The perturbations $\theta_{b}^{\prime}, H_{b}^{\prime}$ have real and imaginary parts. The resulting real part turns out to be positive so that $Q_{r}^{\prime}>0$. This means that the maximum $Q$ will be near the crest,
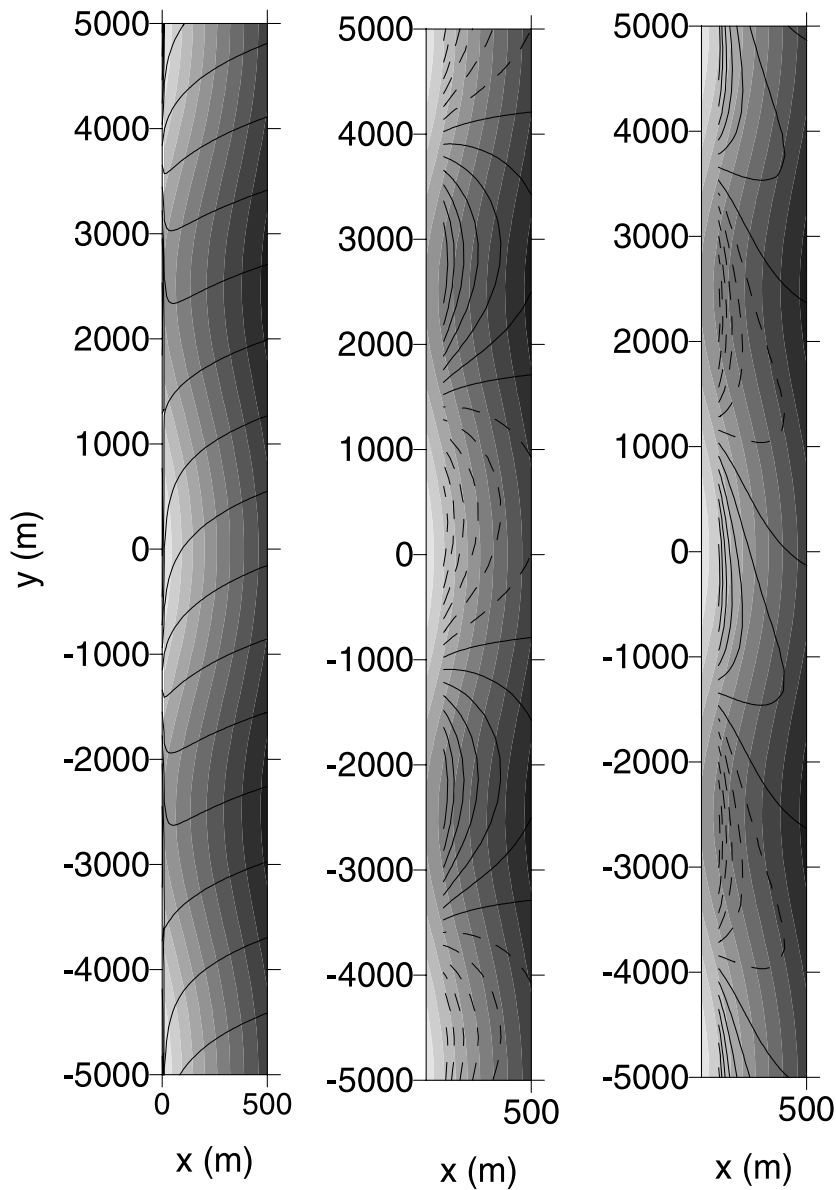

Figure 8. Model run in the "long sand wave limit." (left) Wave fronts. (center) Perturbation in wave angle, $\theta^{\prime}$; positive values are indicated by continuous lines, and negative values are indicated by dashed lines. The bathymetry is indicated by light shading and dark shading for shallow and deep, respectively. (right) Perturbation in wave height, $H^{\prime}$; positive values are indicated by continuous lines, and negative values are indicated by dashed lines. Variable $\lambda=5 \times 10^{3} \mathrm{~m}, \theta_{\infty}=70^{\circ}, s=0.3, L=700 \mathrm{~m}$, and $H_{\infty}=1 \mathrm{~m}$. The cross-shore distances have been magnified by a factor 2 , with the result that the angle between shore and wave fronts on the left panel is larger than the actual one. The wave fronts very close to the shoreline may look somewhat unrealistic due to the arbitrary amplitude of the linearized solution that has been chosen. This does not affect the model results since only the wave direction up to the breaking point is needed.

$-\lambda / 4<y<\lambda / 4$, and hence, the sand wave will migrate downdrift, no matter it grows or decays. Now, for long sand waves, the imaginary parts of $\theta_{b}^{\prime}, H_{b}^{\prime}$ are positive, making $Q_{i}^{\prime}>0$ and tending therefore to produce a maximum transport at a distance $\lambda / 4$ updrift of the horns, just opposite to the term coming from the change in orientation of the coastline. However, for small $L$ and small angles their magnitudes are small and the $i$ term dominates with the result that $Q_{i}^{\prime}<0$, the maximum in $Q$ is in $-\lambda / 4<y<0$ and hence, the sand wave decays while it migrates. By increasing both $L$ and $\theta_{\infty}$ the effect 

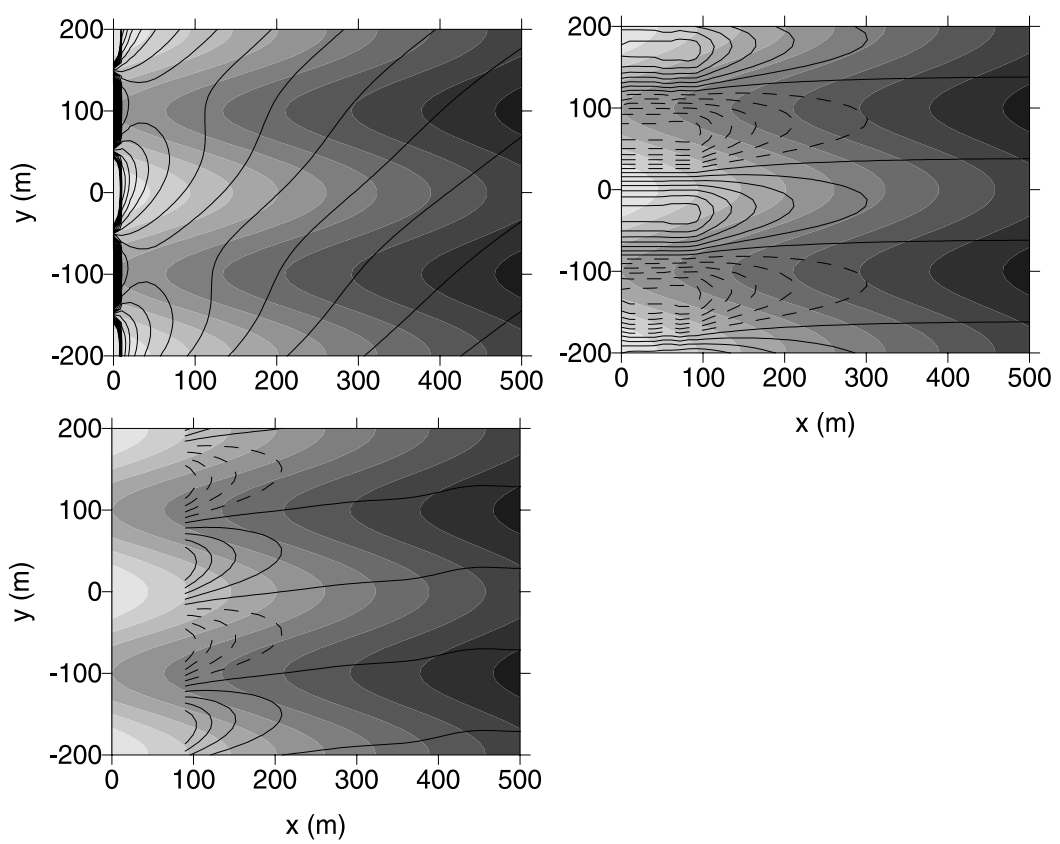

$x(m)$

Figure 9. Model run in the "short sand wave limit." The bathymetry is indicated by light shading and dark shading for shallow and deep, respectively. (top left) Wave fronts. (top right) Perturbation in wave angle, $\theta^{\prime}$. (bottom left) Perturbation in wave height, $H^{\prime}$. Variable $\lambda=100 \mathrm{~m}$, and the rest of the parameters are as in Figure 8. Same comments on wave fronts and positive/negative values as in Figure 8.

of $\theta_{b}^{\prime}, H_{b}^{\prime}$ eventually dominates and the maximum in $Q$ shifts to $0<y<\lambda / 4$ making the sand wave grow.

\subsection{Stabilization of Short Waves}

[38] The stabilization of short waves can also be understood in terms of sediment transport gradients. Figure 8 shows the wave perturbed fronts along with the contour lines of $\theta^{\prime}(x, y)$ and $H^{\prime}(x, y)$ for relatively long sand waves $(\lambda=5000 \mathrm{~m})$. Likewise, Figure 9 displays the same information for very short sand waves with $\lambda=100 \mathrm{~m}$. It is seen in both cases how the maximum refraction (i.e., minimum $\theta^{\prime}$ ) occurs at the lee of the sand wave, consistently with $\theta_{b i}>0$. However, it also becomes apparent that the wave focusing at the lee is much stronger in case of short sand waves than in case of long sand waves. As a result, in case of short waves there is a strong convergence of wave energy downdrift of the horns so that the maximum in wave height shifts from somewhat updrift of the crest (long waves) to about $\lambda / 4$ downdrift of the crest (short waves). The imaginary part of $H_{b}^{\prime}$ becomes therefore negative and overwhelms the imaginary part of $\theta_{b}^{\prime}$ (see equation (33).) Thus the joint effect of both terms plus the $i$ term causes $Q_{i}^{\prime}<0$ (equation (34)) and the maximum $Q$ shifts again downdrift of the crests, the coastline being therefore stable.

\subsection{High Waves Versus Mild Waves}

[39] The instability is favored by small values of $D_{b} / D_{c}$, that is by relatively small waves with respect to the highest waves in the wave climate which are associated to the depth of closure $\left(H_{e} \sim D_{c} / 1.6[\right.$ Komar, 1998]). This is because the bathymetric perturbation extends up to the depth of closure. Then, small waves are affected by this topographic perturbation during a long way up to breaking. In contrast, large waves break very soon after reaching the depth of closure and are hardly affected by the bathymetric perturbation. Our results suggest that instability develops for relatively mild waves and is damped out during severe storms. The same reasoning explaining the influence of the $D_{b} / D_{c}$ parameter explains also the role of $L$. For very small $L$, the bathymetric perturbation is confined close to the coast so that the waves hardly feel it before breaking. There is therefore no HAWI in this case.

\subsection{Influence of the Topography}

[40] The instability also depends on the equilibrium topographic profile, steeper shorefaces being more conducive to instability than gently sloping ones. This has the following explanation. On a gentler shoreface, a shift of the equilibrium profile (see equation (28)) produces a smaller topographic perturbation for a given amplitude of the sand wave than on a steeper one. Even though the topographic perturbation in both cases is gradually reduced in the model to vanish at the depth of closure (equation (29)), still it is true that the offshore extension of the topographic perturbation is much smaller with respect to $x_{c}$ in case of a gentle slope. As a result, in case of steeper shoreface the waves start to feel the topographic perturbation immediately after arriving at the depth of closure while in case of gentler shoreface they still keep on refracting and shoaling on the unperturbed topography a long way before starting to feel the perturbed topography. Therefore the perturbed quantities $\theta_{b}^{\prime}, H_{b}^{\prime}$ are smaller in the latter case and the instability weaker.

\section{Application to the Dutch Coast}

[41] Given the long time- and length scales of the processes described by the model, a quantitative verification against field data is difficult. In this respect, the Dutch coast 


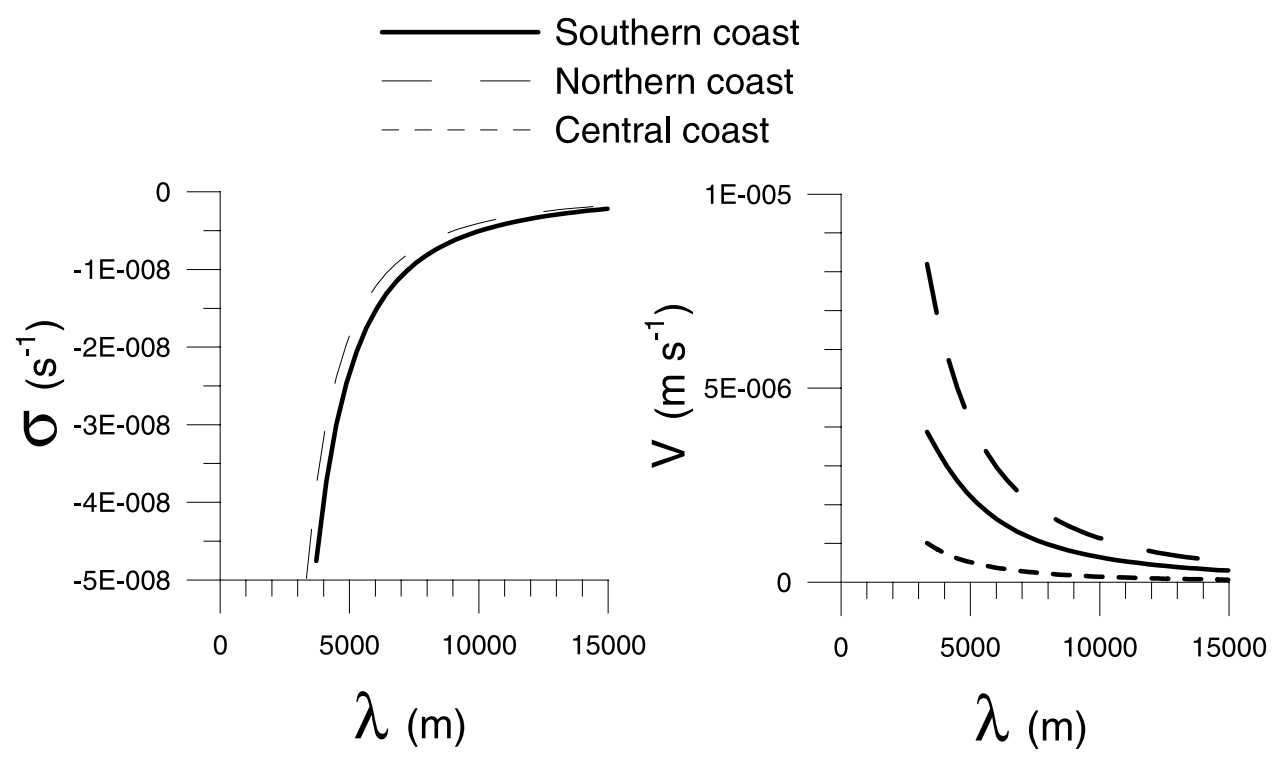

Figure 10. (left) Predicted growth rate and (right) alongshore celerity of the HAWI on the Dutch coast as a function of the wavelength of the coastline waves.

offers however a long sandy coastline whose position has been monitored during the last 150 years. With various techniques, Bakker [1968], Verhagen [1989], Guillen et al. [1999], and Ruessink and Jeuken [2002] have detected, after removing the mean trends, the propagation of waves on the coastline position along this coast. According to Ruessink and Jeuken [2002], the wavelength is about $3.5-10 \mathrm{~km}$ and the amplitudes are small, ranging from $a=20$ to $50 \mathrm{~m}$. The celerity ranges between 0 and $0.2 \mathrm{~km} / \mathrm{yr}$. Looking at Ruessink and Jeuken [2002, Figures 1, 3, and 6], it becomes apparent that on the southern part (Delta coast) there is a clear northward propagation at about $0.07 \mathrm{~km} / \mathrm{yr}$. On the central coast (Holland) this becomes unclear: most of the waves seem to stop, but some of them seem to go on northward whereas some of them seem to propagate southward. It depends on the location and on the time period, southward propagation being apparent only during the last 50 years. This is also consistent with results of Guillen et al. [1999] who analyzed the Jarkus data set during the period 1964-1992 and found southward propagation of sand waves along the Holland coast. Their wavelength is however quite short, $\lambda \sim 2-3 \mathrm{~km}$, and the celerity is $V \simeq 0.15-$ $0.2 \mathrm{~km} / \mathrm{yr}$. In the northern stretch of the coast (Wadden coast), there is again a clear propagation to the northeast with a celerity of about $0.13 \mathrm{~km} / \mathrm{yr}$, which is clearly higher than on the delta coast. The results of the analysis by Verhagen [1989] are qualitatively in agreement with Ruessink and Jeuken [2002] but some quantitative differences arise. Larger sand wave amplitudes ranging from 30 to $500 \mathrm{~m}$ are reported and the wavelengths are larger too, ranging from 2.5 up to $22 \mathrm{~km}$.

[42] The fact that model computations for a generic coast predict HAWI dominant wavelengths in the range $4-15 \mathrm{~km}$ which is in the same range of observed wavelengths along the Dutch coast is certainly intriguing and suggest that these sand waves might be generated by this mechanism. However, a preliminary study by Ashton et al. [2003] indicated that the shore-obliqueness of the wave climate of the Dutch coast is relatively low being at the instability threshold according to their model. An application of our model to the Dutch coast is here shown. For the sake of brevity and since the main purpose of this paper is to present the model and its implications for a generic coast, we will omit most of the details and we will focus only on the main results. The reader who is interested in a more detailed discussion is advised to contact the authors.

[43] The wave climate has been taken from the National Institute for Costal and Marine Management/RIKZ (Golfklimaat web site). For the Wadden coast the ELD station $\left(x_{\infty}=1.5 \times 10^{4} \mathrm{~m}, D_{\infty}=26 \mathrm{~m}\right)$ has been used. The Ijmuiden station $\left(x_{\infty}=3.6 \times 10^{4} \mathrm{~m}, D_{\infty}=22 \mathrm{~m}\right)$ has been used for the central and southern coasts. The incidence angles and significant wave heights have been divided into $20^{\circ}$ and $1 \mathrm{~m}$ intervals. A probability of occurrence $p_{j}$ and a mean wave period $T_{j}$ were assigned to each bin characterized by a $\theta_{\infty}$ and a $\left(H_{s}\right)_{\infty}$ intervals. From the linearity of the governing equation (18), it is readily seen that the resulting growth rate and celerity of a sand wave of a certain wavelength are

$$
\sigma_{r}=\sum_{j=1}^{n} p_{j} \sigma_{r j} \quad V=\sum_{j=1}^{n} p_{j} V_{j},
$$

where $\sigma_{r j}$ and $V_{j}$ are the growth rate and celerity during wave conditions $\left(\theta_{\infty},\left(H_{s}\right)_{\infty}, T\right)_{j}$.

[44] Figure 10 shows the growth rates for $\lambda$ between 3 and $15 \mathrm{~km}$. It is seen that all sand waves tend to decay, any stretch of the coastline being HAWI stable. The southern part is the most stable while the northern part is a little less stable. Typical decay times, e.g., for $\lambda \sim 10 \mathrm{~km}$, are about 8 years. In agreement with Ashton et al. [2003], it turns out that the stability of the coast is due to the insufficient wave obliqueness. Indeed, looking at the wave climate on the southern coast, one of the main wave directions is $220^{\circ}-$ $240^{\circ}$. Model runs with the real wave heights and periods but 
by assuming that all the waves come from the $230^{\circ}$ angle gave instability with a dominant wavelength of $6.5 \mathrm{~km}$ and a growth time of 13 years. Thus the spreading of angles on the real wave climate renders the coast stable.

[45] Figure 10 also shows the migration celerity of sand waves for $\lambda$ between 3 and $15 \mathrm{~km}$. Here, the model results are quite encouraging. Both the sand waves along the Delta coast and along the Wadden coast migrate to the north/ northeast and on the latter they do with a larger celerity. On the central coast they migrate also to the north but with much smaller celerity. In the latter case it is found that small changes in model conditions (e.g., profile of the topographic perturbation) can reverse the migration celerity. This is fully consistent in a qualitative sense with the data analysis of Ruessink and Jeuken [2002]. However, also the quantities are close to observations in case of the southern and the northern stretches of coast. Model predictions are within the correct order of magnitude for the observed range of wavelengths. For instance, in case of $\lambda=7 \mathrm{~km}$, the celerities are $0.039 \mathrm{~km} / \mathrm{yr}$ and $0.071 \mathrm{~km} / \mathrm{yr}$ for the Delta and the Wadden coasts, respectively. However, even a perfect agreement of $0.070 \mathrm{~km} / \mathrm{yr}$ and $0.13 \mathrm{~km} / \mathrm{yr}$ is attained in case of $\lambda=5 \mathrm{~km}$. This is remarkable given the large number of simplifications of the model and the fact that no parameter tuning has been done. For instance, the constant $\mu$ in front of the CERC formula, equation (2), has been fixed to $0.15 \mathrm{~m}^{1 / 2} \mathrm{~s}^{-1}$ which is well in the center of the accepted interval. For the Holland coast the predicted celerity is $0.016 \mathrm{~km} / \mathrm{yr}$ in case of $\lambda=$ $5 \mathrm{~km}$. This small value and the fact that it can be even reversed by changing model conditions is also consistent with observations according to which there is no clear propagation direction along this section of the coast.

\section{Discussion and Conclusions}

[46] The dynamics of small amplitude perturbations of an otherwise rectilinear coastline has been investigated in the framework of an extended one-line model. The main new features of the model are that coastline perturbations are linked to bathymetric perturbations with a finite offshore extend that affect wave transformation from deep water. The latter is done by account of the curvature of the bathymetric lines.

[47] As was discovered by Ashton et al. [2001], the stability or instability of the coast depends on the wave incidence angle $\theta_{\infty}$, larger angles rending the coastline unstable. It is seen however that instability depends on a number of additional factors so that the critical angle for instability is in general higher than the one indicated by that paper $\left(\simeq 42^{\circ}\right)$. It seems therefore that this value is just a lower bound of the critical angle. It is found that instability is favored by sea waves and inhibited by swell. It is also seen that instability is favored by small values of $D_{b} / D_{c}$, that is, by relatively small waves with respect to the highest waves in the wave climate which are associated to the depth of closure. The instability also depends on the equilibrium topographic profile, steeper shorefaces being more conducive to instability than gently sloping ones. A typical timescale for the initial growth of the fastest growing wavelength is in the range of $1-10$ years. This comes out of a continuous forcing with a wave height of $1 \mathrm{~m}$ which is a reasonable value for an annual average rms wave height.
[48] The finding that HAWI always develops with perturbations above some critical wavelength has the very interesting consequence that there is a dominant wavelength. This wavelength is typically in the range $4-15 \mathrm{~km}$ and is proportional to the width of the surf zone, $X_{b}$, with a factor in the order $40-150$. This factor has a relatively complex behavior (specially with $\theta_{\infty}$ ) but in general terms in tends to be larger for small values of $s, D_{b} / D_{c}$ than for large values, that is, for small swell waves rather than for large sea waves. Thus the sand wave wavelength tends to increase with the water wave wavelength. The existence of a dominant wavelength in the linear regime is, in general terms, in contrast with the results of the nonlinear model of Ashton et al. [2001]. In the latter study, there was typically no alongshore characteristic length scale of the shoreline features since the wavelength commonly increased in time simultaneously to the growth in amplitude (except in case of a wave climate consisting of stabilizing and destabilizing waves). On the other hand, that approach adapted to small amplitude perturbations also fails to predict any characteristic length scale of the instability since the governing equation is a diffusion one with a negative diffusivity (see equation (31), where $\partial^{3} x_{s} / \partial y^{3}$ has been disregarded). Thus the present study is clearly in contradiction with the linearized version of that model. The comparison with the nonlinear version is more subtle. On one hand, the present study shows that HAWI do not develop at relatively short length scales. Although Ashton et al. [2001] already suggested that their study was valid only for large length scales, no quantitative lower bound was given. On the other hand, the present study do not contradict the finite amplitude regime described by Ashton et al. [2001] if the associated length scale is large enough. Likely, given a small amplitude perturbation to the coastline, their Fourier components at wavelengths in the range of a few $\mathrm{km}$ can grow. Once the amplitude is significant, the nonlinear cascade toward larger wavelengths described by Ashton et al. [2001] will eventually dominate.

[49] In case of decaying perturbations, even though the governing equation is not a diffusion one, the decay rate provides a useful indication of the coastline diffusivity at the corresponding length scale. In agreement with the simplified approach by Falqués [2003], the diffusivity is typically smaller than its classical linearized prediction [Pelnard-Considère, 1956; Larson et al., 1987; Komar, 1998]. It can be however somewhat larger (up to 10\%) in case of very short sand waves in the order of $2 \mathrm{~km}$ or less. The latter is due to the distribution of wave energy along the sand wave which stabilizes the coastline at short wavelengths. In the limit of large-scale features, Falqués [2003] gives a good prediction in case of sea waves but only the trend in case of swell. It turns out that the classical prediction is very good for large swell waves and moderate angles or, if for whatever reason, the bathymetric perturbation is confined very close to the coast. Of course, when the threshold for HAWI is approached, the classical diffusivity computation for small amplitude features is by no means valid.

[50] The downdrift propagation of coastline waves is not described by the traditional linearized one-line model and the origin of such a propagation is an open problem [see, e.g., Falqués and Calvete, 2003, and references therein]. 
The prediction of this propagation is therefore another interesting result of the present model. It is a consequence of the fact that the refraction and wave energy effects described above do not tend to produce a maximum $Q$ at the point $\lambda / 4$ updrift of the crest but somewhat downdrift of it. However, a detailed analysis of this process and a description of the general results concerning sand wave propagation is beyond the scope of the present paper.

[51] Shoreline cuspate features are sometimes very prominent. This is the case of the capes on the Carolina Coast in USA or the large spits in the Sea of Azov in Ukraine. The finite amplitude dynamics described by Ashton et al. [2001] certainly applies to them. However, shoreline features can also be much more subtle, like the sand waves along the Dutch Coast [Ruessink and Jeuken, 2002; Verhagen, 1989] with wavelength $\lambda \sim 3.5-10 \mathrm{~km}$ and amplitude typically in the range $a \sim 20-50 \mathrm{~m}$. The observed wavelengths fit very well in the range of dominant wavelengths obtained in the present investigation. This suggests that the wavelength selection mechanism presented here could be an explanation for the length scales of shoreline sand waves which are one to two orders of magnitude larger than those of typical surf zone rhythmic features. The model has been therefore applied to the Dutch coast and it has been found that the Dutch coastline forced by the present wave climate is HAWI stable. It turns out that if all waves approached from the SW the rectilinear coastline would be unstable. Thus the stability under the actual climate is mainly caused by the relatively frequent occurrence of low-angle waves. This finding confirms the preliminary results of Ashton et al. [2003]. On the other hand, the model reproduces well the qualitative trends of the sand wave migration along the Dutch coast. Furthermore, even the quantitative predictions are in a reasonable agreement. This indicates that the model captures the main features of large-scale dynamics of sandy coastlines which are diffusivity/instability and sand wave propagation. Turning to the Dutch coast the question still remains about the origin of the observed sand waves. Guillen et al. [1999] suggested that shoreline sand waves along a section of the Holland coast with a wavelength about $2-3 \mathrm{~km}$ could be just forced by surf zone crescentic bars. This is however a very particular case and the wavelength is at the lower limit of those observed. It has also been suggested [Ruessink and Jeuken, 2002] that sand waves could be forced by cyclic behavior of shoals associated to tidal inlets. If this was the case, the Dutch shoreline waves would result from a mixed forced-free behavior: forcing due to tidal inlet morphodynamics and free propagation plus damping induced by the alongshore wavedriven sediment transport as described by the present model. Anyhow, the origin of such sand waves deserves further research.

[52] The main limitation of the present study is the assumption of a fixed cross-shore structure of the topographic perturbation. There is however no other option within the framework of the one-line modeling. In nature, the dynamics of the coastline is linked to the dynamics of the bars, shoals and troughs in the surf zone and beyond it via the longshore and cross-shore sediment transport. The present study disregards all these processes and assumes that if in a certain area there is convergence (divergence) of wave driven alongshore sediment transport this will even- tually result in advance (retreat) of the coastline. Thus the present morphodynamic approach makes sense only on a long timescale (months to years) which is larger than the typical timescale of the dynamics of surf zone bars. This timescale must be large enough for letting the cross-shore beach profile to adjust to equilibrium faster than the sand wave evolution. In particular, the present approach filters out rip currents and the surf zone morphodynamic instabilities which are related to them [Caballeria et al., 2002; Ribas et al., 2003; Reniers et al., 2004]. This is why the short wavelengths at which rhythmic surf zone features appear are damped in the present model.

[53] Finally, another important simplification is that a single wave height, direction and period have been used. The present study can therefore be considered only as a first step toward the most realistic situation of random waves with a probability distribution in height, frequency and angle.

\section{Appendix A: Perturbed Wave Transformation}

[54] The procedure to compute the perturbation of the wave transformation defined by

$$
\begin{gathered}
k=k_{0}(x)+\hat{k}(x)^{\prime} e^{\sigma t+i m y}+\text { c.c. }, \quad \theta=\theta_{0}(x)+\hat{\theta}(x)^{\prime} e^{\sigma t+i m y}+\text { c.c. }, \\
H=H_{0}(x)+\hat{H}(x)^{\prime} e^{\sigma t+i m y}+\text { c.c. }
\end{gathered}
$$

as a function of the topographic perturbation defined in equation (22) is explained in this appendix.

[55] The linearized dispersion relation equation (5) is the algebraic equation

$$
\frac{\hat{k}^{\prime}}{k_{0}}=\frac{2 p}{2 p+\sinh (2 p)} \frac{\hat{h}}{D_{0}},
$$

where $p=k_{0} D_{0}$. The linearized equation (4a) reads

$$
\frac{d S}{d x}-i m\left(\tan \theta_{0}\right) S=-i \frac{m}{\cos \theta_{0}} \hat{k}^{\prime},
$$

where the unknown, $S(x)=k_{0} \hat{\theta}^{\prime} \cos \theta_{0}+\hat{k}^{\prime} \sin \theta_{0}$, is proportional to the phase, $\Phi=-i / m S$. This equation can be solved numerically as an initial value problem with $S\left(x_{\infty}\right)=$ 0 , marching from $x=x_{\infty}$ up to the coastline. Once $\theta(x)^{\prime}$ is known, the linearized equation (4b) can be solved too. By defining

$$
\Psi(x)=2 c_{g} H_{0} \hat{H}^{\prime} \cos \theta_{0}+\hat{c}_{g}^{\prime} H_{0}^{2} \cos \theta_{0}-c_{g} H_{0}^{2} \hat{\theta}^{\prime} \sin \theta_{0},
$$

with $\hat{c}_{g}^{\prime}$ being the perturbation in the group celerity, equation (4b) can be cast into

$$
\frac{d \Psi}{d x}-i m\left(\tan \theta_{0}\right) \Psi=i \frac{m c_{g} H_{0}^{2}}{\cos \theta_{0}} \hat{\theta}^{\prime} .
$$

This equation has the same structure than equation (A3) and is solved with the same scheme from the initial value $\Psi\left(x_{\infty}\right)=0$. Once the perturbed wave transformation is known in all the domain, the breaker line quantities are computed from equations (14) and (16).

[56] Acknowledgments. This work has been carried out during a sabbatical of the first author, which has been partly spent at the University 
of Utrecht (IMAU). This author would like to thank Huib E. de Swart and his Coastal Group for their support and inspiring discussions during that period. Remarks and comments by a number of colleagues are gratefully acknowledged, especially those by Rolf Deigaard. The sabbatical of the first author has been jointly funded by UPC and the Spanish Government under grant PR2003-0440. The second author has been funded by the Spanish Government through the "Ramón y Cajal" program. Thanks are also due to the National Institute for Costal and Marine Management/RIKZ (The Netherlands) for providing information on the Dutch wave climate. This work is part of the HUMOR project, funded by the European Commission under contract EVK3-2000-22014-HUMOR.

\section{References}

Ashton, A., A. B. Murray, and O. Arnault (2001), Formation of coastline features by large-scale instabilities induced by high-angle waves, Nature $414,296-300$

Ashton, A., A. B. Murray, and G. B. Ruessink (2003), Initial tests of a possible explanation for alongshore sandwaves on the Dutch coast, in Proceedings of the IAHR Symposium on River, Coastal and Estuarine Morphodynamics, vol. 3, edited by A. Sánchez-Arcilla and A. Bateman, pp. 320-330, Int. Assoc. for Hydraul. Res., Delft, Netherlands.

Bakker, W. T. (1968), A mathematical theory about sand waves and its application on the Dutch Wadden Isle of Vlieland, Shore Beach, 36, 4-14. Caballeria, M., G. Coco, A. Falqués, and D. A. Huntley (2002), Self-organization mechanisms for the formation of nearshore crescentic and transverse sand bars, J. Fluid Mech., 465, 379-410.

Falqués, A. (2003), On the diffusivity in coastline dynamics, Geophys. Res. Lett., 30(21), 2119, doi:10.1029/2003GL017760.

Falqués, A., and D. Calvete (2003), Propagation of coastline sand waves, in Proceedings of the IAHR Symposium on River, Coastal and Estuarine Morphodynamics, vol. 2, pp. 903-912, Int. Assoc. for Hydraul. Res. Delft, Netherlands.

Guillen, J., M. J. F. Stive, and M. Capobianco (1999), Shoreline evolution of the Holland coast on a decadal scale, Earth Surf. Processes Landforms, $24,517-536$
Horikawa, K. (1988), Nearshore Dynamics and Coastal Processes, Univ. of Tokyo Press, Tokyo.

Komar, P. D. (1998), Beach Processes and Sedimentation, 2nd ed., Prentice-Hall, Upper Saddle River, N. J.

Larson, M., and N. C. Kraus (1991), Mathematical modeling of the fate of beach fill, Coastal Eng., 16, 83-114.

Larson, M., H. Hanson, and N. C. Kraus (1987), Analytical solutions of the one-line model of shoreline change, technical report, U.S. Army Corps of Eng., Washington, D. C.

Mei, C. C. (1989), The Applied Dynamics of Ocean Surface Waves, World Sci., Hackensack, N. J.

Murray, A. B., A. Ashton, and O. Arnoult (2001), Large-scale morphodynamic consequences of an instability in alongshore transport, in Proceedings of the Symposium on River, Coastal and Estuarine Morphodynamics, Obihiro, Japan, vol. 404, pp. 355-364, Int. Assoc. for Hydraul. Res., Delft, Netherlands.

Pelnard-Considère, R. (1956), Essai de theorie de l'evolution des formes de rivage en plages de sable et de galets, in 4th Journees de l'Hydraulique, Les Energies de la Mer, Paris, vol. III-1, pp. 289-298, Soc. de Hydrotech. de Fr., Paris.

Reniers, A. J. H. M., J. A. Roelvink, and E. B. Thornton (2004), Morphodynamic modeling of an embayed beach under wave group forcing, J. Geophys. Res., 109, C01030, doi:10.1029/2002JC001586.

Ribas, F., A. Falqués, and A. Montoto (2003), Nearshore oblique sand bars, J. Geophys. Res., 108(C4), 3119, doi:10.1029/2001JC000985.

Ruessink, B. G., and M. C. J. L. Jeuken (2002), Dunefoot dynamics along the Dutch coast, Earth Surf. Processes Landforms, 27, 1043-1056.

Verhagen, H. J. (1989), Sand waves along the Dutch coast, Coastal Eng., $13,129-147$.

D. Calvete and A. Falqués, Applied Physics Department, Universitat Politècnica de Catalunya, Jordi Girona 1-3, Mòdul B5, Barcelona 08034, Spain. (calvete@fa.upc.es; falques@fa.upc.es) 